\title{
A New Segmentation Algorithm for Knowledge Acquisition in Tissue-Characterizing Magnetic Resonance Imaging
}

\author{
Heinz Handels and Thomas Tolxdorff
}

\begin{abstract}
Tissue-characterizing magnetic resonance imaging (MAI) is a new imaging method for differentiation and biochemical characterization of tissue based on multidimensional MR-parameter information. To support knowledge acquisition in tissue-characterizing MRI, a now segmentation algorithm has been developed by using clustering techniques. The visualizetion of the complex biochemical MR-parameter information is performed by extraction of regions with similer biochemical properties. The clustering algorithm leads to an easy and comfortable handling of the complex tissue-characteristic MR information and supports knowledge acquisition for knowledgebased tissue characterization.

O 1990 by W.B. Saunders Company.
\end{abstract}

KEY WORDS: medical image processing, magnetic resonance imaging, relaxometry, tissue characterization, cluster analysis.

$\mathbf{M}$ AGNETIC RESONANCE imaging (MRI) is a medical imaging method that has opened new possibilities to improve medical in vivo diagnoses. Usually MRI visualizes anatomical structures using only one parameter, the magnetization. By using special multiecho measurement sequences it is possible to analyze tissue-specific relaxation processes in each volume element of a body slice. The relaxation processes, which reflect biochemical tissue properties, are characterized by relaxation parameters. In order to segment tissue types successfully, experience with MRI analysis has shown that it is necessary to analyze combinations of multiple MR parameters, rather than just a single relaxation parameter.

In MRI the usually generated images that are used in the clinical routine are a visualization of the magnetization values $M$ measured in each volume element of a particular slice in the human body (Fig 1). The measured signals $M$ are influenced by several superimposing relaxation processes, which can be characterized by the relaxation times $T_{1}$ and $T_{2}$ as well as the spin density $\rho$. Whereas the relaxation times $T_{1}$ and $T_{2}$ describe the relaxation behavior of the longitudinal and transversal relaxation processes, respectively, the spin density $\rho$ gives information on the density of the spinning protons within one volume element.
The values of the measured signals $M$ depend on the tissue-specific MR parameters $T_{1}, T_{2}$, and $\rho$. Furthermore, the measured signals are also influenced by the experimental parameters TR (repetition time) and TE (echo time), which can be adjusted by the physician.

To describe the relaxation behavior of different superimposing transversal relaxation processes, we use a multiexponential model for the transversal relaxation

$$
\mathrm{M}=\mathrm{M}(0) \sum_{i=1}^{n} \alpha_{\mathrm{i}} \times \exp \left(-\mathrm{TE} / \mathrm{T}_{2 \mathrm{i}}\right)
$$

where TR is constant and $n$ is less than or equal to 3 . The $T_{2 i}$ values correspond to several proton classes in one volume element and $\mathrm{M}(0)_{i}=$ $\mathrm{M}(0) * \alpha_{\mathrm{i}}$ represents the intrinsic magnetization of the $\mathrm{i}$-th proton class at the time zero. The $\alpha_{\mathrm{i}}$ value defined by

$$
\alpha_{\mathrm{i}}=\mathrm{M}(0)_{\mathrm{i}} / \sum_{j=1}^{n} \mathrm{M}(0)_{\mathrm{j}}
$$

describes the partial volume, which is assigned to the $\mathrm{i}$-th proton class in one volume element.

Regarding the additionally occuring $T_{1}$ relaxation process, the measured magnetization is described by

$$
\begin{aligned}
M=c \times \rho \times\left[1-\exp \left(-\mathrm{TR} / \mathrm{T}_{1}\right)\right] & \\
& \times \sum_{i=1}^{n} \alpha_{\mathrm{i}} \times \exp \left(-\mathrm{TE} / \mathrm{T}_{2 \mathrm{i}}\right)
\end{aligned}
$$

whereby $M(0)=c \times \rho \times\left[1-\exp \left(-T R / T_{1}\right)\right]$ and $c$ represents an experimental constant factor.

From the Institut für Medizinische Statistik und Dokumentation, Rheinisch-Westfalische Technische Hochschule Aachen, Aachen, West Germany.

Address reprint requests to Heinz Handels, Institut für Medizinische Statistik und Dokumentation, RheinischWestfalische Technische Hochschule Aachen, D-5100 Aachen, West Germany.

(C) 1990 by W.B. Saunders Company.

0897-1889/90/0302-0002\$03.00/0 


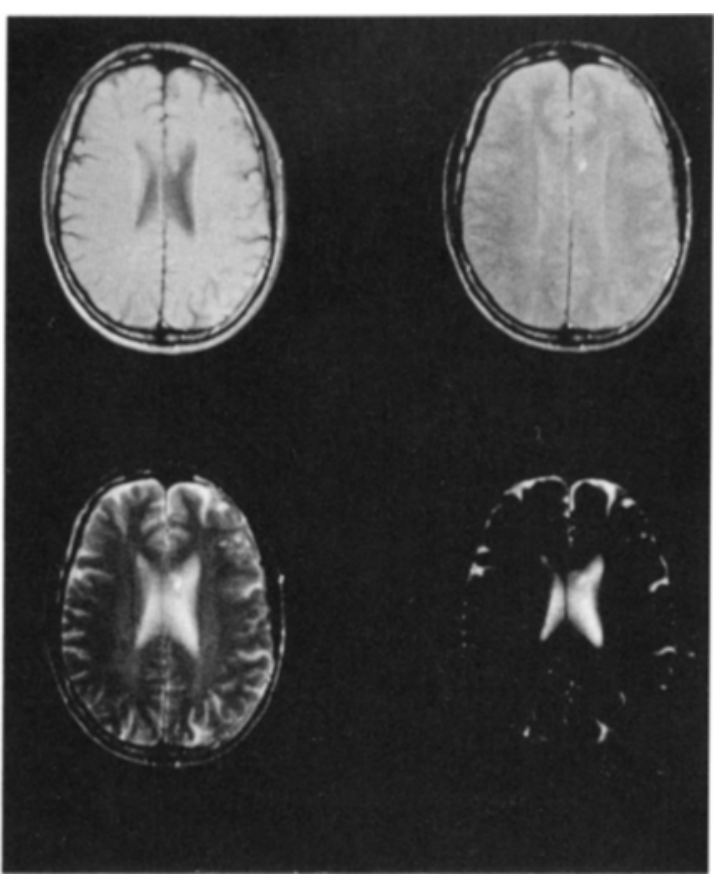

Fig 1. Echo images of one slice at four different echo times TE, (TR $=2$ seconds).

\section{EXPERIMENTAL CONDITIONS}

The MR parameters $T_{1}, T_{2}$, and $\rho$, which influence the signal and the contrast between different tissue in MR images, can only be determined, if the magnetization is measured at different times TE and TR. Multiexponential $\mathrm{T}_{2}$-analysis is only possible if there is a high number of measurement points at different TEs and constant recovery time TR. Based on a multiecho sequence with up to 32 different TEs ( $\triangle \mathrm{TE}=16$ milliseconds) the transverse magnetization decay in each volume element can be recorded (Fig 2). Using nonlinear optimization algorithms ${ }^{1}$ the transverse magnetization decay can be decomposed in up to three exponential functions (Fig 3). For simultaneous recording of the longitudinal $T_{1}$ and transversal $T_{2}$ relaxation processes, we have developed a special multiecho pulse sequence ${ }^{2}$ in cooperation with the Klinik für Radiologische Diagnostik. This pulse sequence uses two excitation pulses $\alpha_{\mathrm{i}}$ (TR1 $=2.1$ seconds, TR2 $=0.5$ seconds) and 32 read-out pulses $\beta_{\mathrm{i}}(\Delta \mathrm{TE}=16$ milliseconds $)$ in one measurement cycle for the $T_{1}$-determination and multiexponential $\mathrm{T}_{2}$-analysis on the base of 28 signals (Fig 4).

\section{SEGMENTATION}

The relaxation times $T_{1}$ and $T_{2}$ and the spin density $\rho$ characterize the tissue-specific relaxation processes that reflect biochemical tissue properties. Therefore, analysis and visualization of the distribution of these tissue-specific parameters of an investigated slice allow the display of anatomical structures as well as the making of qualitative statements on biochemical states of tissue. ${ }^{3}$ In this situation the physician has to respect the multidimensional information in each pixel corresponding to a volume element.

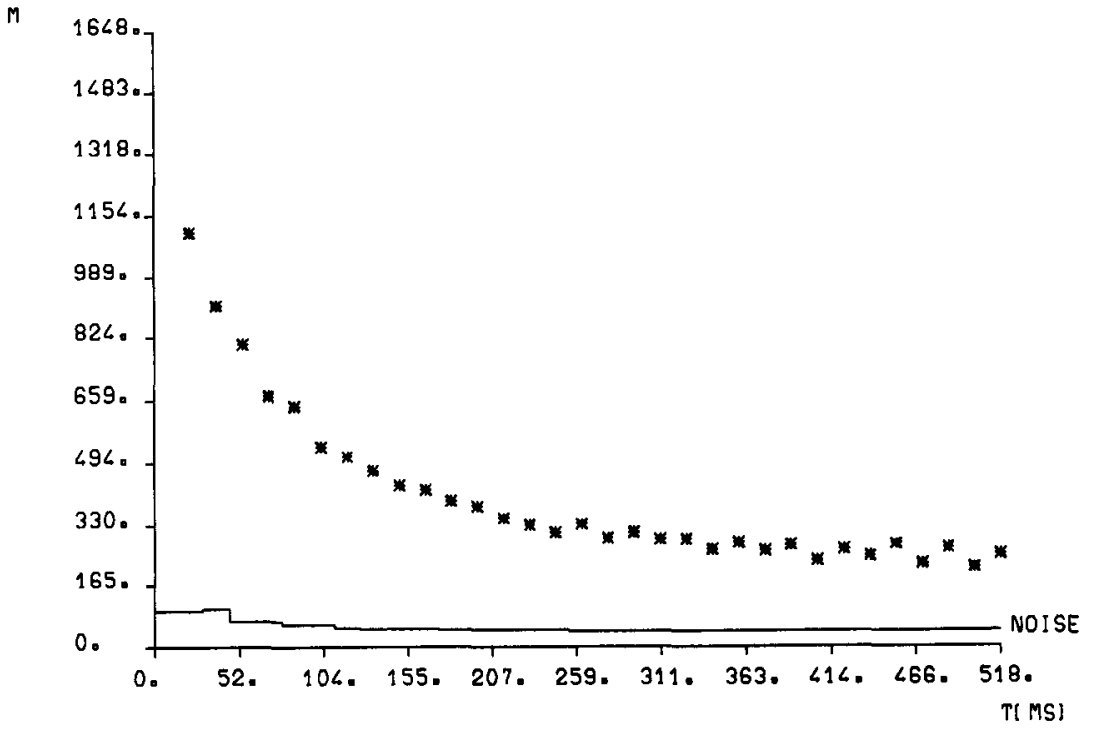

Fig 2. Transversal magnetization decay. 
Fig 3. Decomposition of a multiexponential magnetization decay in three monoexponential components.

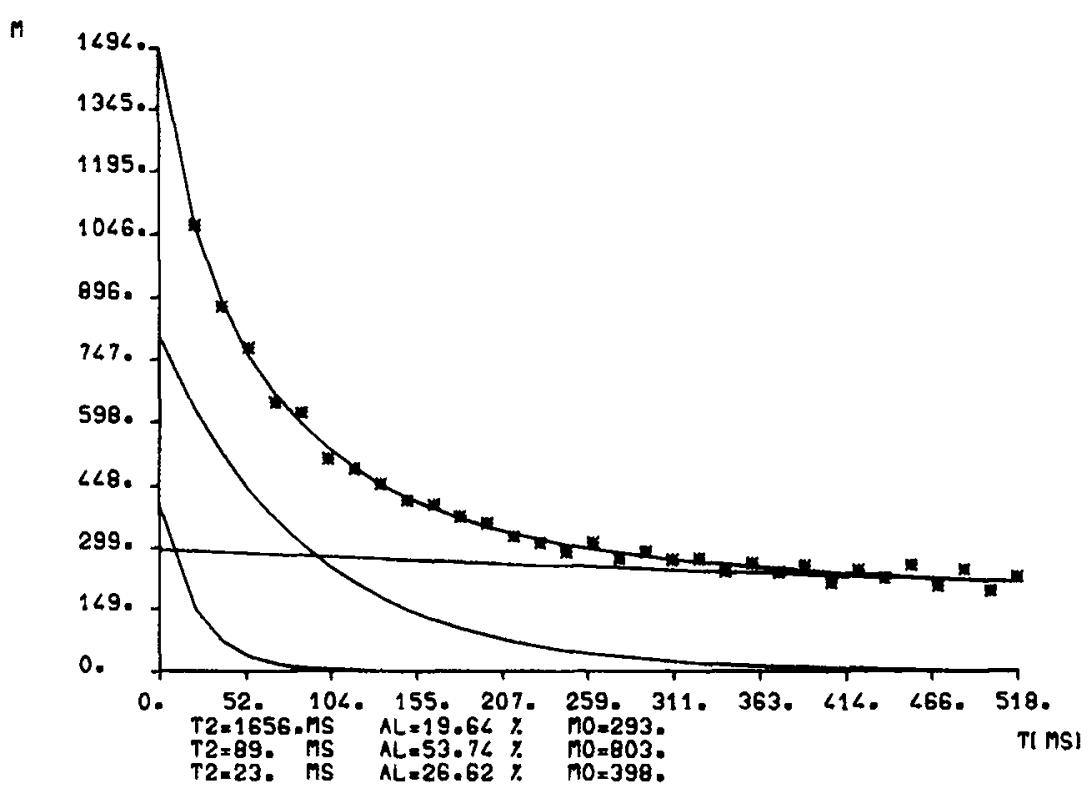

The segmentation is performed by using clustering techniques. First, the physician defines a special region of interest (ROI) within the interested tissue. The ROI, which is described by a $n \times n$ matrix (default: $n=5$ ), can be created easily by positioning a cross-hair in the center of the ROI. This is shown in Fig 5 in the upper left position.

The system computes mean and standard deviation vectors of the one-, two-, and three-dimensional parameter values of $P \in\left\{T_{1}, T_{2}, \alpha, \rho\right\}$ in the considered ROI. Then the following cluster definition is used for imaging. A pixel with $\mathrm{n} \in$ $\{1,2,3\}$ relaxation components and corresponding MR parameter values $\left(P_{1}, \ldots, P_{n}\right)$ belongs to the $P$-cluster $C(P, k)$, if $\forall i=1, \ldots, n$

$$
\left|\overline{\mathrm{P}}_{\mathrm{ni}}-\mathrm{P}_{\mathrm{i}}\right| \leq \mathrm{k} * \sigma_{\mathrm{ni}}
$$

$\mathrm{P} \in\left\{\mathrm{T}_{1}, \mathrm{~T}_{2}, \alpha, \rho\right\} ; \overline{\mathrm{P}}_{\mathrm{ni}}=\mathrm{i}$-th component of the $n$-dimensional mean vector of the parameter $\mathbf{P}$; $\sigma_{\mathrm{ni}}=\mathrm{i}$-th component of the n-dimensional standard deviation-vector of the parameter $P ; k=$ cluster factor.

The clustering algorithm is performed by se- lecting all pixels, which belong to the cluster $C(P, k)$. The selected pixels represent all voxels with a parameter structure similar to the parameter structure of the ROI. The software tool allows us to visualize the multidimensional MR parameter information in different ways, generating color-coded multicluster images as well as simple or combined cluster images.

The results of the segmentation are illustrated by two medical examples. In the first example (Figs 5, 6, and 7) a normal human supraorbital head slice is shown, in which the white matter is segmented. The other segmentation example (Figs 8 and 9) shows an axial human head slice with an astrocytoma, a special kind of brain tumor.

\section{COLOR-CODED MULTICLUSTER IMAGES}

All P-clusters $\mathrm{C}(\mathrm{P}, \mathrm{k})\left(\mathrm{P} \in\left\{\mathrm{T}_{1}, \mathrm{~T}_{2}, \alpha, \rho\right\}, \mathrm{k}=\right.$ $1,2,3,4,5,6)$ can be visualized simultaneously in a so-called color-coded multicluster image (Fig 5). The selection criteria used by the clustering algorithm can be described by the discrete func-

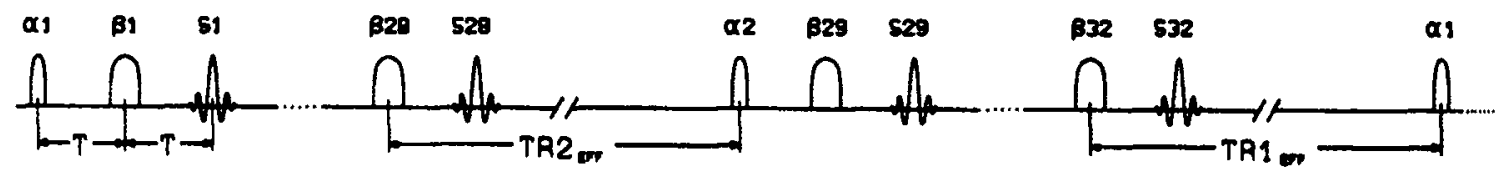

Fig 4. Pulse sequence scheme with two excitation pulses $\alpha_{1}, 32$ read-out pulses $\beta_{1}$, and the measured 32 signals $S_{\text {f. }}$ 


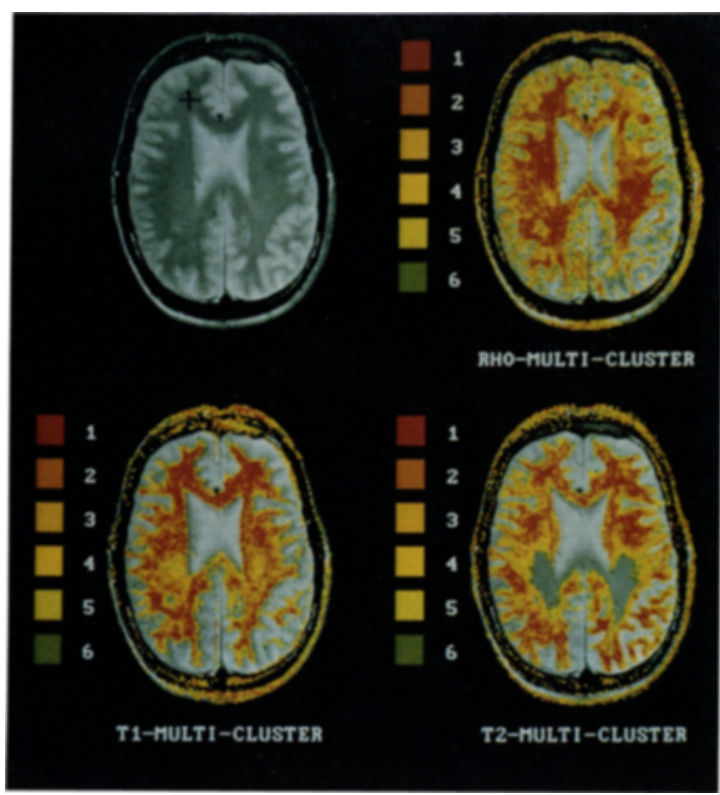

Fig 5. Creation of a ROI in the white matter and multicluster images.

tion $\mathrm{M}_{\mathrm{P}}$ defined by

$\mathrm{M}_{\mathrm{P}}:[\mathrm{X} \times \mathrm{Y}] \rightarrow\{1,2,3,4,5,6,7\}$

with $X=Y=\{0, \ldots, 255\}, x \in X, y \in Y$

$\mathrm{M}_{\mathrm{P}}(\mathrm{x}, \mathrm{y})=\left\{\begin{array}{l}1, \text { if } \mathrm{P}(\mathrm{x}, \mathrm{y}) \in \mathrm{C}(\mathrm{P}, 1) \\ 2, \text { if } \mathrm{P}(\mathrm{x}, \mathrm{y}) \in \mathrm{C}(\mathrm{P}, 2) \backslash \mathrm{C}(\mathrm{P}, 1) \\ 3, \text { if } \mathrm{P}(\mathrm{x}, \mathrm{y}) \in \mathrm{C}(\mathrm{P}, 3) \backslash \mathrm{C}(\mathrm{P}, 2) \\ 4, \text { if } \mathrm{P}(\mathrm{x}, \mathrm{y}) \in \mathrm{C}(\mathrm{P}, 4) \backslash \mathrm{C}(\mathrm{P}, 3) \\ 5, \text { if } \mathrm{P}(\mathrm{x}, \mathrm{y}) \in \mathrm{C}(\mathrm{P}, 5) \backslash \mathrm{C}(\mathrm{P}, 4) \\ 6, \text { if } \mathrm{P}(\mathrm{x}, \mathrm{y}) \in \mathrm{C}(\mathrm{P}, 6) \backslash \mathrm{C}(\mathrm{P}, 5) \\ 7, \text { if } \mathrm{P}(\mathrm{x}, \mathrm{y}) \notin \mathrm{C}(\mathrm{P}, 6)\end{array}\right.$

The clustering algorithm is performed for the spin density $\rho$, the partial volume $\alpha$, and the relaxation times $T_{1}$ and $T_{2}$. Corresponding to the function $M_{p}$ for each $M R$ parameter a colorcoded multicluster image can be generated (Fig 5). Six different color codes of the heat scale are used to represent the six pixel classes 1 to 6 evaluated by the selection algorithm for each MR parameter. The remaining pixels, which are assigned to the seventh pixel, are visualized by the gray values of an echo image. Color-coded multicluster images give an overview about the distribution of distances between the parameter mean-vectors and the parameter values according to each pixel. Furthermore, it is a useful tool to find convenient parameter cluster sizes, which can be used for the generation of cluster images.

\section{CLUSTER IMAGES}

In a cluster image, all voxels of one $\mathrm{T}_{1}, \mathrm{~T}_{2}, \alpha$, and $\rho$ cluster can be displayed using an interactively changeable cluster factor $(k=2$, by default). The selection is described by the Boolean function $S_{p}$ :

$$
\begin{aligned}
& \mathrm{S}_{\mathrm{P}}:[\mathrm{X} \times \mathrm{Y}] \rightarrow\{0,1\} \\
& \text { with } \mathrm{P} \in\left\{\mathrm{T}_{1}, \mathrm{~T}_{2}, \alpha, \rho\right\} \text { and } \mathrm{x} \in \mathrm{X}, \mathrm{y} \in \mathrm{Y} \\
& \mathrm{S}_{\mathrm{P}}(\mathrm{x}, \mathrm{y})=\left\{\begin{array}{l}
1, \text { if } \mathrm{p}(\mathrm{x}, \mathrm{y}) \in \mathrm{C}(\mathrm{P}, \mathrm{k}) \\
0, \text { if } \mathrm{p}(\mathrm{x}, \mathrm{y}) \notin \mathrm{C}(\mathrm{P}, \mathrm{k})
\end{array}\right.
\end{aligned}
$$

The selected voxels are shown in overlay display mode $^{4}$ visualizing anatomical structures (gray values) and biochemical cluster information (color values) in one image (Figs 8 and 9).

To get a specific biochemical characterization of the interested tissue, different parameter selection criteria can be combined to visualize all voxels with a multidimensional parameter structure similar to the ROI. Therefore, the software system allows us to calculate the intersection of generated binary cluster images. The results of this transformation are visualized in combined cluster images (Figs 6 and 7). In the examples, the performed intersections lead to the segmentation of the tissues of interest, the white matter (Fig 6) and the brain tumor, an astrocytoma (Fig 7). The tissues are represented by the colored pixel in overlay display technique.

Based on the voxels of the visualized tissue the calculation of the mean- and standard deviationvectors of the MR parameters $\mathrm{T}_{1}, \mathrm{~T}_{2}, \alpha$, and $\rho$ is performed. These tissue-characteristic values are displayed and stored in a data base to support automatic tissue characterization.

\section{CONCLUSION}

Cluster analysis of MR parameters computed by multiexponential evaluation is a new method to find regions of similar biochemical properties. With this method the spatial distribution of tissue, for example fat, muscle, grey and white matter of the brain, or cerebrospinal fluid, as well as pathological tissue, for example malignant carcinoma of the female breast or astrocytoma in the brain, can be visualized. The software tool described is integrated in the software system 


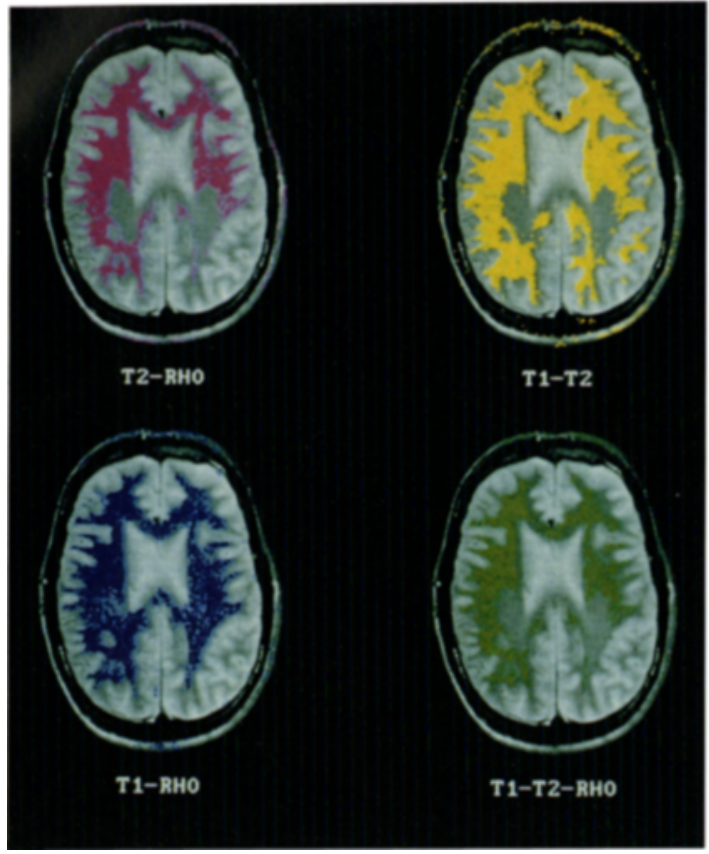

Fig 6. Combined cluster images showing the astrocytoma.

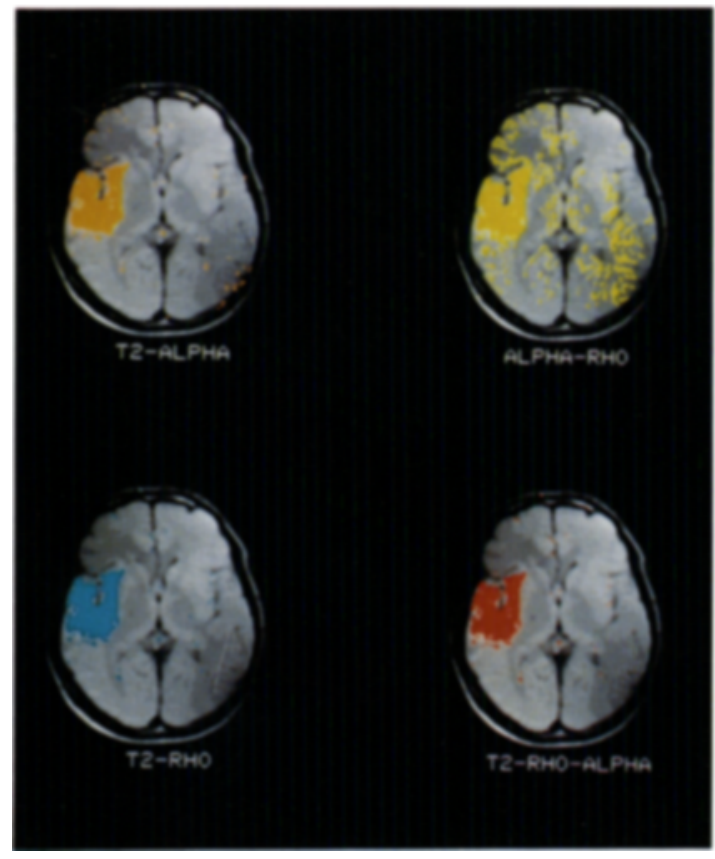

Fig 7. Creation of a ROI in an astrocytoma and cluster images. $T_{1}$ relexation time has not been measured.

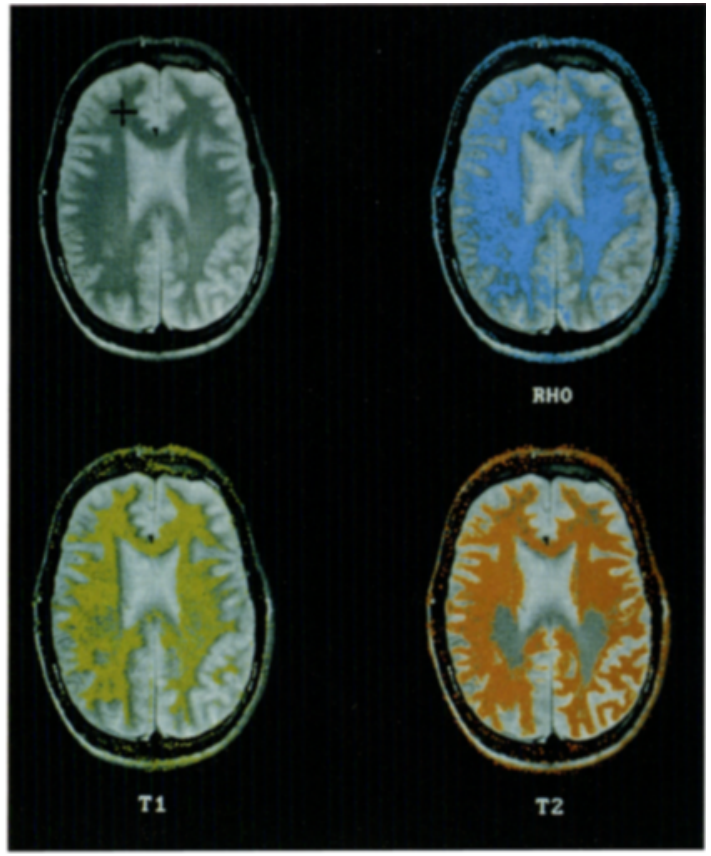

Fig 8. Combined cluster images showing the white matter.

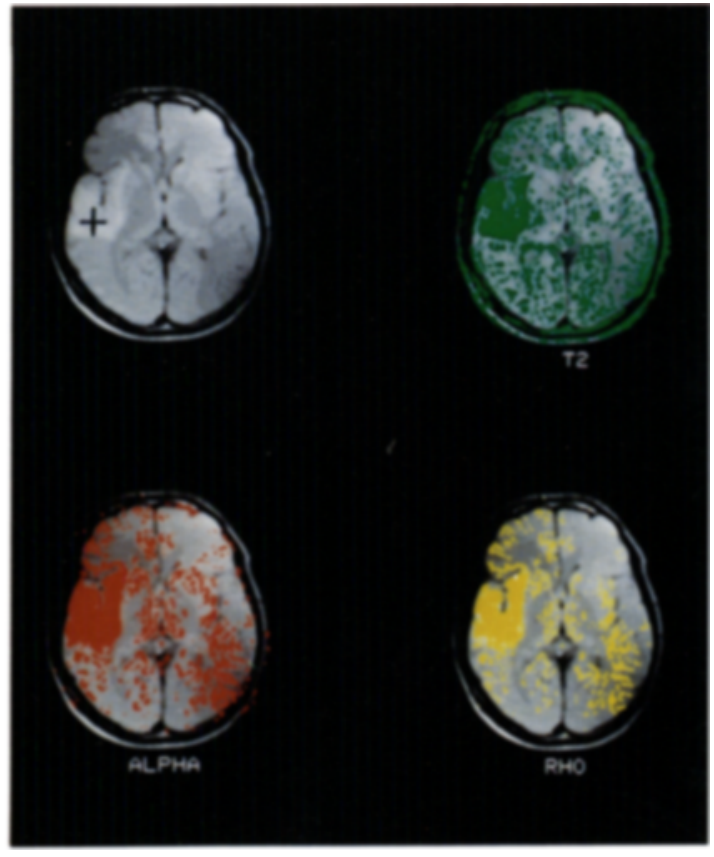

Fig 9. Creation of a ROI in the white matter and cluster images. 
RAMSES (RWTH Aachen Magnetic Resonance Software System) ${ }^{5}$. It enables the physician to use the complex MR parameter information in an easy and comfortable way. Furthermore, knowledge about the coherence between segmented tissue and the multidimensional MR parameter cluster is stored in a tissue data base.

\section{ACKNOWLEDGMENT}

The authors thank Professor Armin Thron from the Department of Neuroradiology and Dr Klaus Bohndorf of the Department of Radiology at the Klinikum of the RheinischWestfälische Hochschule Aachen for making available the MR imagery used in this report. Furthermore, we thank Dipl Phys Manfred Eis for the support of our work by providing the pulse sequence, which has been used for image generation.

\section{REFERENCES}

1. Repges R, Tolxdorff T, Felsberg L, et al: Decomposition of multi-exponential transverse magnetization decays in $\mathrm{T}_{2^{-}}$ selective imaging employing the subsystem EVALUATE of RAMSES, in Lemke HU, Rhodes ML, Jaffee CC, et al (eds): Computer Assisted Radiology, Computerunterstützte Radiologie. New York, NY, Springer-Verlag, 1985, pp 25-29

2. Eis $\mathbf{M}$, Handels $\mathrm{H}$, Bohndorf $\mathrm{K}$, et al: A new method for combined $T_{1}$-measurement and multi-exponential $T_{2}$-analysis in tissue characterizing MRI. Proc 8th Annual Meeting of the Society of the Magnetic Resonance in Medicine, Amsterdam, The Netherlands, 1989, p 770

3. Gersonde K, Tolxdorff T, Felsberg L: Identification and characterization of tissues by $T_{2}$-selective whole body proton NMR-imaging. Magn Reson Med 1:390-401, 1985

4. Tolxdorff T, Felsberg L, Gersonde K: Proton NMRimaging: Contrast-enhanced images and combination with parameter-selective images by overlay display technique, in Lemke HU, Rhodes ML, Jaffee CC, et al (eds): Computer Assisted Radiology. New York, NY, Springer-Verlag, 1987, pp 3-11

5. Tolxdorff T: Ein neues Software-System (RAMSES) zur Verarbeitung NMR-spektroskopischer Daten in der bildgebenden Diagnostik. Medizinische Informatik und Statistik 66. New York, NY, Springer-Verlag, 1987 